\title{
HIV Testing for PMTCT in Tanzania: Time to move from 'Voluntary' to 'Mandatory'?
}

Kahabi Isangula

Aga Khan University, kahabi.isangula@aku.edu

Audrey Holmes

Griffith University

Sharon Brownie

Aga Khan University, sharon.brownie@aku.edu

Follow this and additional works at: https://ecommons.aku.edu/eastafrica_fhs_sonam

Part of the Nursing Commons

\section{Recommended Citation}

Isangula, K., Holmes, A., Brownie, S. (2015). HIV Testing for PMTCT in Tanzania: Time to move from 'Voluntary' to 'Mandatory'?. Advances in social sciences research journal, 2(2), 131-138.

Available at: https://ecommons.aku.edu/eastafrica_fhs_sonam/90 


\title{
HIV Testing for PMTCT in Tanzania: Time to move from 'Voluntary' to 'Mandatory'?
}

\author{
Kahabi Ganka Isangula \\ The Johns Hopkins Bloomberg School of Public Health \& Triangle Solutions, Tanzania
}

Audrey Holmes

Griffith University, Social and Population Health Program, Gold Coast Australia

\author{
Sharon Brownie \\ Griffith University Gold Coast, Australia \& Oxford PRAXIS Forum \\ Green Templeton College, Oxford University, UK
}

\begin{abstract}
Introduction: Every year, many infants are infected with HIV, mostly in sub-Saharan Africa. This is predominantly attributed to mother-to-child or "vertical" transmission during pregnancy, labor and delivery, and breastfeeding. Advances in antiretroviral therapy (ART) and funding have made the prevention of mother-to-child transmission (PMTCT) of HIV more affordable in sub-Saharan Africa. However, despite this advance and its potential in PMTCT, the uptake of HIV testing among pregnant women as an entry point to PMTCT services remains unsatisfactory in many countries. Methods \& Results: In the present paper, authors' present a viewpoint that supports mandatory HIV testing for pregnant women and argue that a mandatory HIV testing policy should be adopted in Tanzania. The ongoing debate about implementing mandatory HIV testing for all pregnant women is discussed in terms of a parental obligation towards protecting a newborn child. Evidence for mandatory HIV testing in prisons (e.g., for prostitution related crimes and sexual offenses), as well as mandatory pre-marriage testing is considered. The way in which the legal framework in Tanzania could support mandatory testing is discussed. Conclusion: Authors' highlight how a national policy of mandatory HIV testing will increase the enrollment of pregnant women into PMTCT services, minimize the risk of HIV transmission to newborn children, improve health outcomes for both parents and children, and contribute to reducing the burden on limited health resources.
\end{abstract}

Keywords: HIV Testing, PMTCT, Voluntary, Mandatory, Tanzania

\section{BACKGROUND}

HIV testing for pregnant women is the optimal entry point to prevention of mother-to-child transmission (PMTCT) services, especially when HIV is diagnosed during the early stages of the antenatal period. More recently, Globally, HIV testing has been moving from client-initiated "opt in" (voluntary testing and counseling) or routine testing to provider-initiated testing and counseling, with the right to refuse or "opt out" as a scale-up and gateway to PMTCT services (1). With an opt out approach, it is expected that HIV testing is more difficult to avoid and greater numbers of women find out about their HIV status, meaning more informed choices about appropriate care can be made (2). However, many countries continue to face poor enrollment into PMTCT programs. In part, this can be attributed to poor uptake of HIV testing as the first step of entry into care as a result of being voluntary or having an opt out option. Poor enrollment into PMTCT services therefore follows the low uptake of HIV testing, particularly in resource-limited settings. New policy approaches must be considered to address this issue. 
HIV/AIDS is a serious disease that has high health costs for pregnant women and infants. In pregnant women, HIV increases the chances of infections that may lead to unfavorable pregnancy outcomes. In addition, the immature immune system of a newborn makes the infant highly susceptible to the consequences of HIV/AIDS illnesses, particularly the opportunistic respiratory infections common in infants less than 6 months of age (3). The health costs are even higher for infants born to a mother who is unaware that she is HIV positive, and is therefore not receiving treatment. HIV-infected newborns have a much-reduced life expectancy (about 2 years) and a reduced quality of life, including an increased risk of various life threatening illnesses (2). A further potential harm if a HIV-infected mother does not access PMTCT services is the risk of AIDS-related death for her and her partner, making the infant one of a growing number of AIDS orphans and increasing the burden on already strained resources.

Access to HIV screening in early pregnancy means that women with HIV and their infants are able benefit from appropriate and timely interventions such as antiretroviral medications, scheduled cesarean delivery, and alternatives to breastfeeding (4). Antiretroviral (ART) prophylaxis or treatment helps to boost a pregnant woman's immune system and allows her to complete her pregnancy normally. This forms a foundation for the first argument about benefits of mandatory HIV Testing for Pregnant women. Nicholson (5) comments that most advocates for mandatory HIV testing reason that "the detection of HIV in a pregnant woman is a first step in getting both her and her fetus on the treatment protocol so as to protect the mother's health and hopefully prevent the transmission of HIV to the baby before, during (and soon after) child birth" (p.175). HIV testing will therefore help to identify those who should receive ARV prophylaxis or treatment for the benefit of both infant and mother.

The introduction of mandatory HIV testing has also been debated in view of the obligation of parents to protect an infant. However, human rights activists, the Center for Disease Control and Prevention, and the World Health organization embrace policies in which providerinitiated routine HIV testing processes explicitly state the right of an individual to refuse or opt out (6). While this position may have initially been based on the global view that a woman has a right to authority over her own body, it does not take into account the obligation of both pregnant women and health authorities to reduce the number of children born with HIV, particularly in countries or areas where there is access to preventative care and treatment (2).

In Tanzania, in 2009, over 1.6 million women aged 15-49 years were estimated to be pregnant. In the same year, 86,000 pregnant women in the same age range were estimated to have tested positive for HIV (7). However, in the same period, the number of children (0-14 years) estimated to be living with HIV was 160,000; twice the number of women who tested HIV positive (8). In a study in southern Tanzania, Harms et al. (9) found that the awareness and knowledge of mother to child transmission and preventive methods was $67 \%$, and that although the "expressed acceptance" of HIV testing was greater than $90 \%$, only $14 \%$ of interviewees had taken a HIV test (pp.262-264). In 2010, the estimated number of pregnant women in Tanzania estimated to have tested positive for HIV was 114,906 (p.19), meaning that the number of HIV positive pregnant women increased significantly in a one-year period (10).

The Tanzania PMTCT Surveillance Report (10) noted that about 97\% of health facilities that have Reproductive and Child Health ( $\mathrm{RCH})$ services also provide PMTCT services. While this is promising, and an estimated $98 \%$ of pregnant women attended an antenatal care (ANC) clinic at least once, not all were tested for HIV. Of those who attended an ANC clinic at least the four recommended visits, only $47 \%$ were likely to undertake an HIV test, while $10 \%$ will never be 
tested for HIV. In addition, the prevalence of HIV (6.9\%) in those women who attended an ANC clinic who had been tested was much higher than the national HIV prevalence of 5.1\% (10).

Likewise, while $98 \%$ of pregnant women attended an ANC clinic at least once, approximately $50 \%$ of births occurred at home (10). The discrepancy between women attending ANC and facility-based births highlights the need for mandatory HIV testing at ANC clinics to "capture" and make treatment available to HIV positive pregnant women who may otherwise deliver at home. HIV testing at any ANC visit will allow initiation of PMTCT services for pregnant women, especially those who may not deliver at the facility, reducing the chances of HIV transmission to infants regardless of the place of birth. This information highlights the need to implement new approaches for HIV testing for all pregnant women, to improve outcomes for mothers and their infants. The low uptake of HIV testing in ANC clinics is not a problem that Tanzania faces alone. In Zimbabwe, only 55\% of women who attended ANC took a HIV test, and 21\% of those who did not take the test, would not have taken it even if there was an opt out option (11).

According to the Tanzanian Ministry of Health and Social Welfare (12), the vision guiding the national health policy is "to improve the health and well being of all Tanzanians with a focus on those most at risk, and to encourage the health system to be more responsive to the needs of the people" (p.4) Given this national health aspiration, mandatory HIV testing for all pregnant mothers is a reasonable public health policy response to infants' need for protection from HIV transmission during pregnancy.

Armstrong (13) noted that there have been proposals for mandatory testing in South Africa. Some researchers report that mandatory testing may be the only promising option, particularly in high-prevalence settings. In most previous cases, mandatory testing has been applied in the context of "low HIV prevalence and a well-resourced health care system" (pp. 24). There is also evidence for mandatory HIV testing for other groups such as travelers to specific countries, pre-marital couples, and newborns of mothers of unknown status. The present paper presents a viewpoint in support of mandatory HIV testing for pregnant women and argues that Tanzania needs to adopt a national approach to mandatory HIV testing. This is a view that has been expressed by a number of other researchers.

\section{MATERIALS AND METHODS}

A review of publically available data was undertaken to identify documents and publications presenting information about PMTCT Services in Tanzania and Africa, as well as evidence for mandatory HIV testing in different settings. A search of relevant reviews and articles was conducted via the internet and included PubMed, BioMed Central, and the databases of agencies such as the World Health Organization (WHO), Tanzanian Ministry of Health and Social Welfare, and a general search on Google Scholar. Combinations of keywords (e.g., Tanzania, PMTCT, HIV Testing, Mandatory) were used to define the search. Due to the limited material available, strict inclusion and exclusion criteria were not set. Abstracts were screened for keyword relevance.

\section{RESULTS \& DISCUSSION \\ Mandatory HIV testing in Prisons and for Sexual offenses}

HIV infection has been reported to be a significant health problem faced by many prisons around the world (14). There is a range of evidence for mandatory HIV testing in the prison contexts (15). Mandatory HIV testing in prisons has been suggested to be an important adjunct to minimizing the impact of the spread of the virus, both within prisons and in the nonoffender population (16). A study conducted in Brazil found that incarcerated sexual offenders with HIV showed a higher recidivism risk that non-infected offenders, and higher general and sexual impulsiveness (14). This highlights an important implication of adopting a mandatory 
testing approach, in that such a policy allows health care providers to intervene early and control the spread of the disease. For example, the AIDS Policy Law journal (17) notes that in the US state of Washington, although the law makes HIV testing voluntary for convicted offenders and pretrial detainees, the test is mandatory for prostitution-related crimes and certain other sex offense convictions. Currently, mandatory HIV testing is not a requirement in prisons in Tanzania, although a test may be required for sexual offenders (HIV and AIDS Prevention and Control Act, 2008)(18). However, experience from other settings may indicate that there is a need for mandatory testing in specific high-risk groups.

\section{Mandatory Pre-marital HIV testing}

Another example of a mandatory HIV testing policy is that of premarital HIV testing. Mandatory HIV testing before marriage is a premarital requirement common in many countries around the world, particularly in places that have strongly faith-based organizations $(19,20)$. However, mandatory premarital testing is a topic of debate as it is considered an infringement of the fundamental human rights of those who are infected and a possible cause of social stigma. Despite the global controversy, mandatory premarital HIV screening remains a practice in Tanzania. Arulogun (19) reports that $82.8 \%$ of youth believed that mandatory premarital HIV testing could reduce the spread of HIV, about $52.6 \%$ did not think it would increase stigma, and the majority had a positive attitude toward mandatory premarital testing. Premarital couples often agree to form a relationship through a decision making process. The couple, their families, and at times their wider community are included in this decision making process, and even if it is not a religious requirement, the decision to test for HIV is often reached based on individual obligatory responsibility of protecting one another.

\section{Ethics and Human Rights in Mandatory HIV Testing}

Debates about mandatory HIV testing for pregnant women frequently focus on the human rights of a pregnant woman, including her right to autonomy and privacy. However, a point that much of the research literature fails to articulate is that in pregnancy, a fetus does not have opportunity to decide whether to be born to a particular mother. This raises the question that if couples have an obligation or responsibility to protecting one another from HIV infection, why would mother's responsibility to protect an innocent infant (who did not have a choice about being born) be unethical?

Globally, only $7 \%$ of WHO member states report having legislative measures for HIV prevention to address testing pregnant women in the prenatal period, and only $9 \%$ have set measures requiring quarantine, isolation, or coercive hospitalization of HIV-infected people or AIDS patients (21). Some governments restrict people with HIV/AIDS from entering their countries with the aim of reducing HIV transmission, although HIV-related border restrictions is considered to be a violation of the human right of freedom of movement $(22,23)$.

According to MMWR (4), if a mother's HIV status is still unknown after birth, CDC guidelines recommend rapid antibody testing of newborns as soon as possible to allow for immediate initiation of antiretroviral prophylaxis. If the infant tests positive in antibody tests, it implies that the mother is positive, and efforts are made to confirm this and enroll her into care. The unanswered questions in much of the literature are: Is it a newborn's responsibility to protect mother's health? Should we wait for the newborn to disclose its mother's status? Or, should we test a mother as a parental obligation to protect the newborn? This last question is vital, as a newborn's tests do not conclusively show the baby's HIV status, as all babies born to HIV positive mothers have maternal HIV antibodies. Babies who are uninfected do not lose their 
antibodies until 18 months of age, and the majority of babies with HIV antibodies are actually uninfected by the virus.

A PCR test that detects the actual presence of viral DNA can also be done, but cannot give an accurate result until a baby is at least 6 weeks old. Moreover, babies found to have HIV antibodies would be given antiretroviral prophylaxis, which may prevent HIV developing, but may also potentially have detrimental effects in the long term because of its toxicity.

\section{A Legal Perspective on Mandatory HIV Testing for Pregnant Women}

Around the world, law courts are often confronted with the issues surrounding the conflict between the rights of a pregnant woman, and the prevention of harm to the unborn child. This is further complicated by a commonly held viewpoint that an infant only acquires legal status on being born alive. However, in some parts of the US, there is a developing legislative framework that criminalizes prenatal harm through actions such as alcohol abuse and illegal drug use $(2,27)$.

The risk of HIV transmission begins during pregnancy and continues through breast-feeding. Early detection of the mother's HIV status will provide opportunity for early initiation of PMTCT services, and consequently significantly reduces MTCT. If a mother were to object to this, she is therefore "intentionally" transmitting HIV to an infant, and risks legal action according to the Tanzania HIV/AIDS Prevention and Control Act (18), which requires a person with HIV to protect others from infection (Part VIII: 33:2:1 (a)).

\section{Legal Justifications for Mandatory HIV Testing in Tanzania}

Therefore, the key document that justifies mandatory HIV testing for pregnant women in Tanzania is the Tanzania HIV and AIDS Prevention and Control Act (18). While this legislation prohibits compulsory HIV testing, is also removes the legal protection for people required to take the test under court order, for those donating human organs and tissue, and for sexual offenders. The Act also details the rights and obligations of persons living with HIV and AIDS and requires these people to protect others from infection (Part VIII: 33:2:1 (a)). As HIV is mainly transmitted through unprotected sexual intercourse, pregnancy itself is also a result of unprotected sexual intercourse. Thus, a pregnant woman rejecting an HIV test can be considered HIV positive until proven otherwise, particularly where there is a prior history of high-risk behavior (herself or her partner).

Section 15:8 (b) of the Act authorizes medical practitioners to conduct an HIV test when they "believe that such test is clinically necessary or desirable in interest (of a mother and her infant)", giving a legally justifiable mandate for HIV testing at ANC and labour and delivery clinics in Tanzania. As an innocent infant is involved, knowing if a pregnant woman is HIV positive or not, especially when there is evidence of prior history of risky behavior of a mother or her partner, HIV testing should not be a voluntary option.

While health care workers are supposed to "do no harm", the Tanzania HIV/AIDS Prevention and Control Act (18) mandates medical professionals to conduct HIV testing if they believe that it is reasonably desirable for the benefit of a mother and a growing infant. In addition, the Act mandates a pregnant woman with a parental obligation to "do no harm" and "protect" the baby. This makes mandatory HIV testing professionally as well as legally justifiable in ANC and labour and delivery clinics in Tanzania.

In China, providers were most likely to endorse mandatory HIV testing for patients with highrisk behavior and for all patients before surgery, with over $43 \%$ of providers endorsing mandatory testing for anyone admitted to hospital. Controlling for demographics, multivariate 
analyses indicated that providers with higher perceived risk of HIV infection at work, higher general prejudicial attitudes toward people living with AIDS, and previous contact with HIV patients were more likely to endorse mandatory HIV testing for anyone admitted to hospital (24).

As chances of post-exposure prophylaxis among health care workers are high as a result of poor working conditions (medical doctors' reasons for strike in Tanzania are predominantly poor working conditions (25), mandatory HIV testing is justifiable. From a legal and ethics perspective, an individual's right to privacy and informed consent is certainly violated when HIV testing is something that is required. However, the flip side is when an infant could be spared the infection if the mother's status is revealed. In Tanzania, a low skilled birth attendant rate $(47 \%)$ makes it difficult to protect exposed infants, particularly as about $53 \%$ of pregnant women that attend ANC at least once in the early stages of pregnancy are not tested for HIV and deliver their babies in non health care settings.

Infants can contract the virus through breastfeeding as well as during childbirth. However, in Tanzania, exclusive breastfeeding for infants less than 6 months old is 41\% (10), meaning that breastfeeding is an unreliable and unpredictable method of protecting exposed infants from acquiring HIV through breastfeeding. Early detection of HIV during the antenatal period therefore remains the golden opportunity, As if the proper preventative measures are taken during the antenatal period, that child's life has the potential to be free from HIV, despite its mother's seropositive status.

\section{CONCLUSION AND RECOMMENDATIONS}

HIV transmission risks begin during pregnancy and continue through breast-feeding. Early detection of mother's HIV status will provide opportunity for early initiation of PMTCT services, consequently significantly reducing mother to child transmission (MTCT) of HIV. Newborn tests do not conclusively show the baby's HIV status, as all babies born to HIV positive mothers have maternal HIV antibodies. Babies who are uninfected do not lose their antibodies until 18 months of age and the majority of babies with HIV antibodies are actually uninfected by the virus. A PCR test that detects the actual presence of viral DNA can also be conducted, but cannot give an accurate result until a baby is at least 6 weeks old. HIV testing of pregnant women is therefore the best option to curb uncertainties surrounding HIV exposure in newborns. Mandatory HIV Testing may be the only way to achieve increased HIV test uptake, increased enrolment in PMTCT, and increased protection for new-borns against MTCT.

Although mandatory HIV testing is justifiable in view of the evidence presented here, there several considerations. A key issue is the sustainable supply of test kits. A study in Mwanza, Tanzania, found that although all health care facility staff received HIV testing training, only $60.7 \%$ of the facilities had readily available HIV test kits (26). Logistical issues in the procurement and supply of HIV test kits, currently centralized at the Medical Stores Department needs to be resolved before mandatory HIV testing can be implemented. In addition, post-test issues such as sustainable ARV treatment, treatment of opportunistic infections, and addressing social and other barriers need to be resolved in order to meet the service demand once the mandatory testing is institutionalized.

In conclusion, although it may be difficult for a mother to face the reality of mandatory HIV screening, the question we should ask ourselves as public health officials is: Would the benefit of protecting an infant from contracting the virus not outweigh the shame or stigma that may accompany such action? 


\section{Competing interests}

The authors declare that they have no financial or non-financial competing interests.

\section{Authors' Contributions}

Isangula, $\mathrm{K}$ contributed the initial manuscript concept, accessed local data, provided substantive local insights and interpretation and was the lead writer of the article. Brownie, $S$ and Holmes, A assisted by expanding the literature review, undertaking data interpretation and was the secondary contributing author while also providing editorial advice and review of developing drafts.

\section{References}

Bolu, O, Allread ,V, Creek, T, Stringer ,E, (Eds). (2007). Approaches for scaling up human immunodeficiency virus testing and counseling in prevention of mother-to-child human immunodeficiency virus transmission settings in resource-limited countries. American Journal of Obstetric and Gynecology; 197(3 Suppl): S83-9. Review

Schuklenk, U., \& Kleinsmidt, A. (2007). Rethinking Mandatory HIV testing during pregnancy in areas with high HIV prevalence rates: Ethical and policy issues. American Journal of Public Health, 97(7); 1179-1183.

Simmons, R.J et al. (1993). Pneumocystis Carinii Pneumonia among US Children with Perinatally Acquired HIV Infection, 270 JAMA 470, 470-73.

MMWR. (2006). 'Revised recommendations for HIV testing of adults, adolescents, and pregnant women in healthcare settings', 55;1-17.

Nicholson, E (2003). Mandatory HIV Testing Of Pregnant Women: Public Health Policy Considerations And Alternatives, Duke J. of Gender L. \& Pol'y 175.

Rennie ,S., Mupenda, B. (2008). Ethics of mandatory premarital HIV testing in Africa: the case of Goma, Democratic Republic of Congo. Dev World Bioeth.8(2):126-37

UNGASS. (2010). 'UNGASS Reporting for 2010: Tanzania Mainland and Zanzibar'. Retrieved from http://www.unaids.org/en/Regionscountries/Countries/UnitedRepublicofTanzania/

UNAIDS. (2010). 'UNAIDS report on the global AIDS epidemic. Retrieved from http://www.unaids.org/globalreport/Global_report.htm

Harms, G, Schulze, K, Moneta, I et al (2005). Mother-to-child transmission of HIV and its prevention: awareness and knowledge in Uganda and Tanzania. SAHARA J, 2(2): 258-66

MoHSW. (2011). HIV/AIDS/STI Surveillance Report. National AIDS control Program. Report Number 22 . p.19

Perez, F, Zvandaziva ,C, Engelsmann ,B, Dabis , F. (2006) Acceptability of routine HIV testing ("opt-out") in antenatal services in two rural districts of Zimbabwe. Journal of Acquired Immune Deficiency Syndrome, 41(4):514-20

MoHSW. (2003). National Health Policy; United Republic of Tanzania, Retrieved on December 20,2014 at http://apps.who.int/medicinedocs/documents/s18419en/s18419en.pdf

Armstrong, R. (2008). Mandatory HIV testing in pregnancy: is there ever a time? Developing World Bioethics, 8(1):1-10.

Baltieri, AB, \& Boer, DP. (2011). Do HIV-infected incarcerated sexual offenders deserve a little more attention? The Open Forensic Science Journal, 4, 25-29

Perucci, CA, Michelozzi, P, Arcà, M. (1991). Mandatory test for HIV infections in prison population: evaluation of the efficacy of a screening program. Epidemiol Prev. 13(46): 28-36

Amankwaa AA, Amankwaa LC, Ochie, CO (1999). Revisiting the debate of voluntary versus mandatory HIV/AIDS testing in U.S. prisons. J Health Hum Serv Adm.,22(2):220-36

AIDS Policy Law, (1997). Washington adopts measure for testing prison inmates, 12(11): 4-5.

Tanzania HIV and AIDS prevention and Control Act. (2008). Retrieved on August 9, 2014 at http://www.saflii.org/tz/legis/num_act/haapaca2008336.pdf.

Arulogun, OS, Adefioye, OA. (2010). Attitude towards mandatory pre-marital HIV testing among unmarried youths in Ibadan northwest local government area in Nigeria. African Journal of Reproductive Health, 14(1):83-94 
Isangula K. G., Holmes, A., \& Brownie, S. (2015). HIV Testing for PMTCT in Tanzania: Time to move from 'Voluntary' to 'Mandatory'? Advances in Social Sciences Research Journal, 2(2), 131-138.

Uneke, CJ, Alo, M, Ogbu, O. (2007). Mandatory pre-marital HIV testing in Nigeria: the public health and social implications. AIDS Care, 19(1): 116-21.

D'Amelio, R, Tuerlings, E, Perito, 0, et al. (2001). A global review of legislation on HIV/AIDS: the issue of HIV testing. Journal of Acquired Immune Deficiency Syndrome, 28(2):173-9

AIDS Health Promotion Exchange, (1993). Border restrictions and HIV / AIDS: a public health policy disaster, (1): 12-3.

De Cook, KM, Fowler, et al. (2000). Prevention of Mother -to- Child HIV Transmission in resource poor countries: Translating research into policy and practice. JAMA,283:1175-82.

Li, L, Wu, Z, Wu, S, Lee, SJ et al. (2007). Mandatory HIV testing in China: the perception of healthcare providers. International Journal of STD AIDS, 18(7): 476-81.

Isangula, K, G. (2012). Viewpoint: Moral and Ethical Dilemmas During Medical Doctor's Strike in Tanzania. Negotiation Processes \& Communications eJournal Vol. 4, Issue 12. URL: http://dx.doi.org/10.2139/ssrn.2129024.

Mashauri, FM, Siza, JE, Temu et al.(2007). Assessment of quality assurance in HIV testing in health facilities in Lake Victoria zone, Tanzania. Tanzania Health Research Bulletin, 9(2):110-4

O'Grady , B, Schüklenk, U. (2009). Rethinking mandatory HIV testing. Bioethics. ,23(8):ii. 